\title{
Designing a wireless sensor with ultra-capacitor and PV microcell for Smart Building Energy Management.
}

\section{Fernando del Ama Gonzalo*}

School of Engineering.

American University of Ras Al Khaimah.

Ras Al Khaimah, UAE. PO Box: 10021.

Email: fernando.gonzalo@aurak.ac.ae

* Corresponding author

\section{Belen Moreno Santamaria}

School of Aeronautics and Space Engineering, Polytechnic University of Madrid. Spain.

Email: belen.moreno@upm.es

\section{Juan A. Hernandez Ramos}

School of Aeronautics and Space Engineering, Polytechnic University of Madrid. Spain. Email: juanantonio.hernandez@upm.es

\begin{abstract}
:
Building owners face a number of challenges, as they search for ways to reduce energy consumption, lower operating costs, and manage modern technology and systems. A research group from Aerospace School of Madrid has addressed these aims by developing a real-time Building Management System that consists of a plurality of transmitter nodes, a central controller, and a user interface. Monitoring, control, and actuation systems lack reliable and cost effective data logging and measuring devices of energy parameters in buildings. In this paper, a new concept of wireless sensors is recommended, powered by PV microcells and electric double layer capacitors. With these power source, the sensor can send data every minute without being affected by the absence of sunlight. The feasibility of such a sensor was studied empirically and the findings are reported in this paper.
\end{abstract}

Keywords: Wireless sensors, Ultra-capacitor tests, Smart Home Energy Management.

Reference to this paper should be made as follows: del Ama Gonzalo, F., Moreno, B., Hernandez Ramos J.A. (2017) 'Designing a wireless sensor with ultra-capacitor and PV microcell for Smart Building Energy Management', Int. J. Embedded Systems, Vol. X, No. Y4, pp.000-000.

Biographical notes: Fernando del Ama Gonzalo obtained his PhD in the field of Architecture at Polytechnic University of Madrid. Spain, 2005. He has been an Associate Professor and Chair of Department of Architecture at American University of Ras Al Khaimah, UAE since 2016. His research interest focus on energy management in buildings.

Belén Moreno is Senior Researcher at School of Aeronautics and Space Engineering, Polytechnic University of Madrid. Spain. She completed her studies of architecture in the Technical School of Architecture of Madrid. Her professional career has been enriched in the field of Research and Development of new technologies focused on Energy Efficiency in building at National and International level.

Juan Antonio Hernández Ramos is an Associate Professor of Aerospace Engineering at Polytechnic University of Madrid, Spain. His research experience is related to numerical simulation and high order numerical schemes for thermo-fluid-dynamic problems. 


\section{Introduction}

Over the past few years, most countries around the world have developed new rules and strategies to reduce energy costs in buildings. Since home appliances account for $42 \%$ of all electricity consumed in households, effective energy management systems are considered to be a promising energy saving technique with significant commercial potential. Wireless sensor networks (WSNs) are being established for sensing and actuating critical applications, like industrial automation, domotics and remote patient monitoring (Schrickte, Luiz Fernando et al. 2016).

A typical system consists of one or more transmitter nodes that send measurement data to a receiver or base connected to a host PC, which monitors or records the data. In a star network, one or more transmitter nodes are connected to a centralized receiver. Transmitter nodes cannot communicate directly with each other; they only communicate with the receiver. In a mesh network, all transmitter nodes can communicate with each other. Transmitter nodes can move data between themselves until the data reaches the intended location (Shuo Li et al. 2014).

Figure 1 shows schematic views of both mesh and star wireless sensor network system.

The research group at Aerospace School of Madrid developed a Smart Home Energy Management System to monitor power consumption and identify the operations of home appliances. A central controller interacts with a user interface on an electronic device. In addition to the central controller, the system comprises of mesh network transmitter nodes connected to all kind of home electric devices and a load controller for controlling and monitoring the building electric load. It is compatible with traditional meter reading methods, hence enables remote access for existing energy meter by the energy consumer. They can also monitor the readings of meter regularly and access data stored on the cloud. It is a bidirectional scalable system that automatically recognizes the inclusion of new transmitter nodes. It also shows, at each instant, the status of all devices in the installation. Finally, it allows radio frequency remote control from anywhere in the world, with any kind of internet access while preserving the option of manual control. In order to provide the central controller with Temperature and Relative Humidity values, the research group has tackled the task of designing and manufacturing wireless sensors. In the field of sensors, and in particular wireless sensors, greater integration has been slow because of the difficulties encountered in the integration of microcontroller, RF transceiver, and sensor elements in the one integrated sensor device. These difficulties have arisen from incompatibilities of materials processing for the various elements. The ability to connect meters, or by extension any battery operated end nodes such as wireless sensors, requires a system-level approach combining analog and digital hardware with software expertise.

Sensors integrated into structures, machinery, and the environment, coupled with the efficient delivery of sensed information, could provide tremendous benefits to the society. Sensors and controls have demonstrated a potential to reduce building energy consumption by $20-30 \%$ (Savchuk et al. 2016). Bundles of wires are subject to breakage and connector failures. Long wire bundles represent a significant installation and long term maintenance cost, limiting the number of sensors that may be deployed, and therefore, reducing the overall quality of the data reported. Wireless sensing networks can eliminate these costs, by eliminating connectors and easing the installation process. Low-cost and non-invasive temperature and relative humidity sensors using wireless technology have been developed (Yamauchi et al. 2016). Achieving accurate data requires the ability to integrate sensors, radio communications, and digital electronics into a single integrated circuit package. This capability facilitates the operation of networks with very low-cost sensors that can communicate with each while using low power wireless data routing protocols. Xihai Zhang et al. (2017) found that the positions of nodes are important for environmental sensing. A key feature of any wireless sensing node is the minimum power consumed by the system and the need for electricity storage. A system where batteries have to be changed constantly is likely to experience poor adoption rate. By combining smart sensors and PV microcells, long lasting and maintenance free systems can be made, while eliminating wires and batteries (Churchill et al. 2003).

According to Guo, et al (2012) and Wang et al (2012), electronic products ranging from cell phones and laptops to the electronic systems in spacecraft ought to be small, lightweight and flexible. To this end, Schlapbach and. Zuttel (2001) claim that a lot of effort has been made toward discovering new materials that meet the above requirements. On the other hand, solar energy systems are based on a solar collector, designed to collect the sun's energy and to convert it into either electrical power or thermal energy (Eda et al. 2008; Peng, et al. 2009 and Shaowu et al. 2014). Such systems have emerged as viable sources of renewable energy over the past decades (Raasakka, 1999 and Algifri and Al-Towaie, 2001) and are now widely used for a variety of industrial and domestic applications (Jensen, 2011). According to Kim and Chou (2013), employing an ultracapacitor, a battery or a combination of both as power sources for a system is the best way to store harvested energy. Selection of the battery or ultra-capacitor is usually based on energy or power density, number of chargerecharge cycles, leakage current, and complexity of the charging circuit.

The device proposed in this project reduces the cost barriers of deploying advanced sensors in buildings to enable optimization of energy usage. The wireless sensor is networked and scalable, consumes very little power, is software programmable, capable of fast data acquisition, reliable and accurate over the long term, costs little to purchase and install, and requires no real maintenance. It takes measurements of Temperature and Relative Humidity every 60 seconds. The source of energy is a PV micro cell that provides a microcontroller with electricity. The mean 
intensity in the microcontroller is $0.06 \mathrm{~mA}$. Data is sent without a battery and without any cables. PV microcells are the energy source for the wireless device. The sensor is operatively connected to a microprocessor with programmable sensor data acquisition capabilities, capable of monitoring, and transmitting environmental data to a remote data collector.

\section{Description of the sensor and tests.}

Wen and Smith (2002) found that managing energy consumption in buildings is key to achieving Zero Energy Buildings (ZEB). Nowadays, there is a technology gap in wireless sensors for reliable self-powering mechanisms. The proposed device addresses the gap by developing wireless technology and system-level integration to enable solar powered wireless sensors with significant range to reduce networking infrastructure requirements. The device has been designed to suit Building Management System (BMS) projects. The aim of BMS is to control relevant parameters in buildings such as energy consumption, temperature, humidity, and electric lighting. Several devices monitor these parameters and transmit data to a controller. Figure 2 shows the architecture of the proposed device. It acts as a wireless thermostat and humidity sensor. Data is transmitted via radio. It also offers the possibility of controlling other devices via software, and power is supplied by micro PV cells. The main challenge is to reduce the power consumption.

Wilson (2005) found that the largest power consumption is attributable to the radio link itself. Radio transmission power, transmission cycle time, and ambient conditions effect the power consumption. There are a number of strategies that can be used to reduce the average supply current of the radio. These strategies include reducing the amount of data transmitted through data compression and lowering sensor sample rate to the minimum rate required by the application. The microcontroller has a number of functions including managing data collection from the sensors, performing power management functions, interfacing the sensor data to the physical radio layer, and managing the radio network protocol. This sensor requires little current and low voltage, which can be supplied by a PV panel. At night, an ultra-capacitor is needed to allow the sensor to keep on sending data. By doing so, the ultracapacitor becomes the main power source during the high load states. Then, over the daylight hours, the PV cells will be the main power source and will charge the ultracapacitor. Since the voltage of PV panels depends on the solar irradiance, a Zener voltage regulator is needed to keep the microcontroller safe.

During this project, some tests were conducted to understand the actual performance of the sensor. All tests were aimed at checking correct performance under different conditions. The first tests were carried out on a device that included a battery. Through these tests, the necessary surface of PV panels was determined to harvest energy for a specific period of time. At the same time, an ultra-capacitor was dimensioned to accumulate this energy. The second tests showed that the addition of an ultra-capacitor in parallel with a battery is the most effective choice that a designer can make to maximize battery capacity utilization in low power Radio Frequency applications.

\section{Testing a device without ultra-capacitor.}

In the first test, the voltage was measured when the device was connected to a constant charge. Two resistors were selected to produce a $20 \mathrm{~mA}$ and $13.6 \mathrm{~mA}$ charge. Figure 3 shows the voltage curve. Since a constant charge was connected, voltage and current evolved in similar ways. A resistor was selected to produce $20 \mathrm{~mA}$ and $13.6 \mathrm{~mA}$ charge. It can be observed that there is a performance improvement for currents below $15 \mathrm{~mA}$.

Theoretically, with a nominal capacity of $210 \mathrm{mAh}$ and a current flow of $20 \mathrm{~mA}$, a battery should last around 10.5 hours. Nevertheless, it can be observed that voltage drops below the Smart Reset Radio voltage after 1 hour and 45 minutes. Even when taking into account that Smart Reset Radio voltage is $1.95 \mathrm{~V}$, most optimistic estimations would give 6 hours before reaching $0 \mathrm{~V}$. This is $40 \%$ less than the expected duration of 10 hours. Thus, batteries do not have an acceptable capacity when working under these conditions. On the other hand, with $13.6 \mathrm{~mA}$ of current flow, a battery should last around 15 hours and 27 minutes. In this plot, the battery has been working for 20 hours. Obviously, such increase in battery life is caused by the reduction in current over time, which reduces consumption. Nevertheless, the voltage falls below the Smart Reset Radio voltage after 7 hours.

\section{Election of the ultra-capacitor}

Generally, capacitors have dielectric between two opposite electrodes. For example, Aluminium Electrolytic Capacitors use an aluminium oxide film, and Tantalum Capacitors use a tantalum oxide film as dielectric. However, the electric double layer capacitor or ultra-capacitor does not have a dielectric. It uses a physical mechanism that generates an electric double layer which performs the function of a dielectric, hence, the name Electric Double Layer Capacitor. The charge-discharge factor in the element of electric double layer capacitors is an ion absorption layer, which is formed on the surface of the positive and negative electrodes of activated carbon, to utilize absorptiondesorption reactions. Of late, ultra-Capacitors are in the spotlight as a hybrid power supply system in combination with the solar cell. The ultra-Capacitor is meant to be used in providing constant energy to the sensor at night. In this section, some preliminary tests are described to characterize how the different components work.

Ultra-capacitors are lightweight and can store more energy in a given volume than aluminium electrolytic capacitors. Unlike rechargeable batteries, ultra-capacitors can be recharged hundreds of thousands of times. Unfortunately, ultra-capacitors tend to have significant higher self-discharge rates. That is, they cannot store energy for as long a period as a battery. The aim of this section is to 
test different ultra-capacitors to fit the charge and discharge time to the sensor specifications.

\subsection{Self-discharge}

Manufacturers of ultra-capacitors provide the following equations for the discharging characteristics, which are just an application of general theory of capacitors.

Equation 1 shows the relationship between electric charge, Capacitance and voltage.

$$
Q=C V
$$

Where $\mathrm{C}$ is the capacitance of the capacitor $(1.0 \mathrm{~F}), \mathrm{Q}$ is the electric charge and $\mathrm{V}$ is the voltage between terminals. The same expression in incremental changes is shown in equation 3:

$$
C=\frac{d Q}{d V}
$$

To calculate the capacitance of the capacitor, the following formula is used:

$$
\Delta Q=C \cdot \Delta V
$$

The Ohm's law relates current and electric charge as:

$$
I=\frac{d Q}{d t}=\frac{d V}{d t} \mathrm{C}
$$

The self-discharge characteristic of an electric double layer capacitor is shown in equation 5 .

$$
V=V_{0} \exp \left(\frac{-t}{C \cdot R L}\right)
$$

Where $\mathrm{V}_{0}$ is the initial voltage and $\mathrm{R}_{\mathrm{L}}$ is the insulation resistance.

The time required for the constant current and constant resistance discharging respectively are presented in equation 6.

$$
t=-C R \operatorname{Ln}\left(\frac{V_{1}}{V_{0}}\right)
$$

Where t: Charging time; C: Capacitance; $\mathrm{V}_{0}$ : Initial voltage; $\mathrm{V}_{1}$ : Terminal voltage after $\mathrm{t}(\mathrm{s})$; I : Constant current load; R: Constant resistance load.

In order to validate the abovementioned equations, four capacitors were tested to measure the voltage between terminals. Manufacturers show different curves based on the charging duration; they have 1, 100 and 200 hours charged capacitors. The differences in the self-discharge are very significant, for example a difference of 500 hours more to go under $2.0 \mathrm{~V}$ in the terminals.

In the first approximation, the time of charging and $\mathrm{V}_{0}$ were the parameters we needed to control for the selfdischarge, where $\mathrm{R}_{\mathrm{L}}$ and $\mathrm{C}$ are the main properties of the component. $\mathrm{V}_{0}$ of the sensor was limited to $3.3 \mathrm{~V}$ because the nominal voltage could not be higher, even when the capacitor was charged at 5.5V DC.

With this conclusion, the next test would prove if the charging time is, in fact, important or just influential only when the charging time is around hundreds of hours. In this test, there were four capacitors, all of them charged at around $3.307 \mathrm{~V}$. All were charged individually with the same current over the defined time to ensure that they had the same conditions. Later, the current that each capacitor needed was tested. Capacitor 1 charged for 15 minutes at $3.304 \mathrm{~V}$. The second one for 30 minutes at $3.305 \mathrm{~V}$, and the third one for 11 hours and $3.308 \mathrm{~V}$. The last one was charged for 1 hour and $3.307 \mathrm{~V}$. Figure 4 shows the values for each capacitor.

These results show that even when the charging time is 11 hours, the differences in the voltage are not so big. Capacitor 3 started the first 30 hours with less leaks, but then its behaviour became similar to the others. The objective of this capacitor is to provide energy to the sensor over the night time. It has to be charged over the day time. 11 hours of charge is probably near the limit of artificial light time and charge.

Another interesting result we could obtain is the Insulation Resistance. The curves can be compared to an exponential function, and the coefficient of $\mathrm{t}$ (time) in the exponent can be used to obtain $\mathrm{RL}(\mathrm{C}=1.0 \mathrm{~F})$. After doing this approximation, the Insulation Resistance was 3.6M $\Omega$.

\subsection{Zener + Diode influence.}

In the following tests, three of the capacitors were alone, and one of them had the configuration with a Zener diode and another diode, as shown in Figure 5.

First tests show that the charging time was higher when the capacitor had the circuit shown above. The next tests were carried out using a DC signal generator that could provide constant voltage and enough current for a lot of capacitors at the same time. The sensor was connected to the terminals of the capacitor, and the voltage during the charge was monitored. Different tests were carried out.

In test A, we established that the DC generator supplied 3.364V. It was expected that, within a given time, the capacitor would accumulate the same voltage. However, the charging time duration became very long, yet the maximum voltage the capacitor achieved was just $2.914 \mathrm{~V}$. It is important to note two things; the oscilloscope had a $3.3 \mathrm{~V}$ saturation and there was a difference in the measurements between the oscilloscope and the multimeter Agilent U1232A (of around $0.15 \mathrm{~V}$ ). The oscilloscope makes an average measure each 1.26 second, and uses 1.82 second sending information and calculating. According to the test, 1 measure equals 3.08s. The ultra-capacitor needs 12.8 minutes to be almost fully charged.

Test $\mathrm{B}$ aimed at proving the performance when the voltage was lower than the needed and lower than the nominal voltage of the Zener Diode. The voltage was therefore set to $3.034 \mathrm{~V}$ and Figure 6 shows the results. In 
this test, the charge process was faster - only 5 minutes $(100$ measures approximately). The maximum charge was $2.6 \mathrm{~V}$ when the DC generator was charging at $3.034 \mathrm{~V}$. As it was noticed in test $\mathrm{A}$, there was a difference between the oscilloscope measurements and the measurement taken with the multimeter.

Test $\mathrm{C}$ failed to provide any reliable data because the maximum current that the Zener can withstand was exceeded. Zener Diode BZX79C3V3 from NXP manufacturer was tested. Its maximum power is $500 \mathrm{~mW}$. In the case of $3.3 \mathrm{~V}$, the maximum current will be $0.151 \mathrm{~A}$ or $151 \mathrm{~mA}$

Test $\mathrm{D}$ gave the approximate time needed to charge the capacitor at $3.3 \mathrm{~V}$ in the case that there is enough current but no Zener and no Diode; just the capacitor. The final result was 4 minutes and 5 seconds with no resistors connected and the capacitor was charged to $3.337 \mathrm{~V}$.

\subsection{Resistor discharge}

The goal of this test was to characterize the discharge of the ultra-capacitor using a resistor connected to the device. With a resistor, the discharge process takes less time and the results can be compared with the specifications provided by the company.

The three characteristic currents are $50 \mathrm{~mA}$ for the transmission peak, $300 \mu \mathrm{A}$ for the sensor consumption and $5 \mathrm{~mA}$ when the MCU and the radio are activated, and the MCU is ordering the measurements. Thus, we established $3.3 \mathrm{~V}$ for the test, even when the voltage was decreased continuously. Different charge times were set so that different tests were carried out for each charge time. One capacitor was provided with the Zener and the other one was tested without it. With these currents, the resistance should be, $66 \Omega, 11000 \Omega$ and $660 \Omega$ respectively. The manufacturer recommends $1 \mathrm{~mA}$ as the discharge current.

The first discharge test was carried out with the following values for Resistance and Voltage: $R=1000 \Omega$ and $\mathrm{V}_{0}=3.320 \mathrm{~V}$ (3.3mA maximum). Figure 7 shows tests for the four capacitors: capacitor $\mathrm{A}$ and $\mathrm{F}$ were charged for 5minutes; the first one without Zener and the second one with it. Capacitor B and $G$ were tested in the same conditions without and with Zener, but the charge time was extended to 10 minutes. The charge time does not have an important effect, much less with only 5 minutes of difference. The capacitors that had the Zener and the diode did become fully charged. With only 5 minutes from the start of the measurement, they did not reach $2.0 \mathrm{~V}$.

Capacitor $\mathrm{A}$ and capacitor $\mathrm{F}$ were tested again with the following values for Resistance and Voltage: $R=11000 \Omega$ and $V_{0}=3.331 \mathrm{~V}$ and the results were the same. Figure 8 shows the results of this test considering that the time of charge was 5 minutes. The case with $\mathrm{R}=62 \Omega$ depicts a very quick discharge. In the first minute, the voltage was under $1 \mathrm{~V}$. The sensor device only needs that current for approximately $1.34 \mathrm{~ms}$. Following the experimental results, ultra-capacitor $\mathrm{A}$ is compared to an exponential function in which the exponent is $-0.006 \mathrm{t}$ (time in minutes). Therefore, the resistor value is $10,000 \Omega$. This value matches the specifications provided by the manufacturer.

Using equations (2), (3) and (4) and using average values:

$\int_{V_{0}}^{V_{f}} C d V=\int_{t_{0}}^{t_{f}} I d t$

Considering that consumption is constant, then there are no peaks, and the resulting calculation of the average current is

$I_{a v}=\frac{50 \cdot 1.34+0.3 \cdot 90+5 \cdot 4}{60000}=1.9 \cdot 10^{-3} \mathrm{~mA}$

Developing equation 7 and considering current constant in time, we can express:

$C\left(V_{f}-V_{o}\right)=I_{a v} \cdot\left(t_{f}-t_{0}\right)$

In this example the following values were chosen: $\mathrm{C}=1.0 \mathrm{~F}, \mathrm{~V}_{\mathrm{f}}=2.1 \mathrm{~V}$ and $\mathrm{V}_{0}=3.3 \mathrm{~V}, \mathrm{t}_{0}=0$ : And the result for the final time:

$$
t_{f}=175 \text { hours }
$$

After testing different ultra-capacitors, self-discharge values matched the values provided by the manufacturer in the product data sheet. It is also important to know properly the electrical performances of the Zener to design the most efficient schematic to have a full charge of the capacitor. The Zener has to be protected with a resistance - the Zener's impedance. Finally, the voltage drop of the diode has to be taken into account in order to have a full charge of the capacitor.

\section{Testing a device with ultra-capacitor.}

In order to improve the effective-capacity of the battery, a $220 \mu \mathrm{F}$ ultra-capacitor was connected in parallel with the battery. In this test, voltage and current consumption of the device were obtained. The capacitor provided the battery with a constant current. Even after 100 hours, the average consumption remained around $0.7 \mathrm{~mA}$. Figure $9 \mathrm{a}$ shows the battery's average voltage and average consumption during the test. Over the first test, the device was running at $100 \mathrm{~ms}$ cycle. The ultra-capacitor improved the performance. The battery still supplied enough power after 100 hours and the consumption remained stable. A second test was carried out as shown in Figure 9b. Some components were added: 10K pull up resistor and a $100 \mathrm{nF}$ capacitor for MCU's RESET line in addition to the $220 \mu \mathrm{F}$ capacitor. No evidence of malfunctioning was found in current tests. There were no unexpected transmission peaks, and this means the MCU did not reset randomly.

MCU and RF current consumption are the main challenges in designing wireless sensors. The tests carried out in this section show that adding a capacitor in parallel 
with the rest of the components is the most effective way through which a designer can maximize battery capacity utilization in low power RF applications.

\section{Testing the PV microcell.}

The next design challenge was how to integrate the efficiency of solar energy collection under dynamic light conditions. Adding a capacitor to miniaturized photovoltaic modules can help the device to adjust dynamically to the light intensity variations. Some tests have been carried out to verify the sustainability of sensors powered by solar energy (Brunelli et al. 2008).

Small photovoltaic PV modules have been recently proposed to enable perpetual operation of wireless sensor networks. Over the past few years, Jiang, Polastre and Culler (2005) presented several solutions to supply a sensor node, using a small PV module. Ultra-low power systems designed for wearable devices and smart materials and equipped with photovoltaic harvester have been studied by Ward et al (2006). Simjee and Chou (2006) have focussed on wireless sensor systems that use an ultra-capacitor as energy reservoir.

The next step was to test the capability of different PV microcells to provide the sensor with enough power during day time. The sensor was also tested to make sure that it can operate without sun light due to the energy harvested in the ultra-capacitor.

Figure 10a displays the voltage level of the ultracapacitor used as energy reservoir and which allowed the sensor to operate continuously for about $60 \mathrm{~s}$. Figure $10 \mathrm{~b}$ shows that when the incident light increases, the power generated by the $P V$ module becomes enough for continuous sensor operations and for energy recharge. This test determines the necessary surface of PV panels to harvest energy for a specific period of time. At the same time, an ultra-capacitor can be dimensioned to accumulate this energy.

\section{Future work and improvement.}

The proposed sensor addresses a fundamental issue with many existing devices. Reducing maintenance requirements is key to help the building owners rely on this technology. This aim can be achieved using a combination of photo voltaic micro cells and ultra-capacitors. The components of this sensor were tested individually and the first prototype will be assembled in the near future. The following are possible areas of future work: Lowering system cost and size; increasing operation time; enhancing reliability and integrating the sensor in existing Building Energy Management Systems.

\section{Conclusions.}

Buildings consume up to $40 \%$ of the energy produced in the world. Reducing the cost barrier to deploying advanced building energy management systems is critical in the saving energy consumed in buildings. Ultimately, the system must be able to collect particular environmental data using sensors for instance temperature, moisture, and humidity. Relevant data will be transmitted to a centralized receiver.

The ultimate goal is to achieve a perpetually powered wireless sensor that does not require frequent periodical maintenance and battery replacement, and which recharge more effectively. Experimental measurements have been carried out to determine the design parameters for these wireless devices.

First, the test results on a battery powered device show that using $30 \mathrm{~mA}$ peak current versus $15 \mathrm{~mA}$ peak current increases the battery life, while slightly reducing the effective capacity of a battery.

Second, adding an appropriate ultra-capacitor remarkably improves the effective capacity of the battery. During the testing and validation of module operation, an extensive suite of tests were used to ensure correct and desired functionality. Different tests were carried out in order to select the ultra-capacitor with the required charge and discharge times. Test results met the specified targets from the product datasheets.

Finally, a case study on a real sensor with PV cells demonstrates complete sustainability of the system with solar regenerative energy. Adding an ultra-capacitor ensures that the sensor is continuously supplied with an electrical voltage, thus is able to send regular data at all times, over several years.

\section{Acknowledgement}

This work was supported by program Horizon 2020EU.3.3.1.: Reducing energy consumption and carbon footprint by smart and sustainable use, project ref. 680441 InDeWaG: Industrial Development of Water Flow Glazing Systems.

\section{References}

Algifri, A.H.; Al-Towaie, H.A. "Efficient orientation impacts of box-type solar cooker on the cooker performance”. Solar Energ. 2001, 70, 165-170.

Brunelli, D., Benini, L., Moser, C. Thiele, L. "An Efficient Solar Energy Harvester for Wireless Sensor Nodes". Proceedings Design, Automation and Test in Europe (DATE 08), Munich, Germany, 2008.

Churchill, D.L., Hamel, M.J., Townsend, C.P., Arms, S.W., "Strain Energy Harvesting for Wireless Sensor Networks," Proc. SPIE's 10th Int'l Symposium on Smart Structures \& Materials, San Diego, CA. March, 2003.

Eda, G. Fanchini G, Chhowalla M. "Large-area ultrathin films of reduced graphene oxide as a transparent and flexible electronic material". Nat. Nanotechnol. 3 (2008) 270-274. 
Schrickte, Luiz Fernando; Barros Montez; Romulo Silva De Oliveira, Carlos; Rochildt Pinto, Alex Sandro. "Design and implementation of a 6LoWPAN gateway for wireless sensor networks integration with the internet of things". Int. J. of Embedded Systems, 2016 Vol.8, No.5/6, pp.380 - 390.

Guo, W. et al. "A Novel Electromechanical Actuation Mechanism of a Carbon Nanotube Fiber"Advanced Materials. Vol. 24, Issue 39 (2012) p. 5379-5384.

Jensen, Mathias. "Coin cells and peak current draw" EE Times Europe. November 02, 2011.

Jiang, X. Polastre, J. and Culler, D. E. "Perpetual environmentally powered sensor networks.," in Proceedings of the Fourth International Symposium on Information Processing in Sensor Networks, IPSN 2005, (UCLA, Los Angeles, California, USA), pp. 463-468, April 25-27 2005.

Kim, S.; Chou, P.H. Size and Topology Optimization for Supercapacitor-Based Sub-Watt Energy Harvesters. IEEE Trans. Power Electron. 2013, 28, 2068-2080.

Peng, H.S. et al. "Electrochromatic carbon nanotube/polydiacetylene nanocomposite fibres" Nature Nanotechnology. 4 (2009) p. 738-741.

Raasakka, B. "Solar skylight apparatus". Renew. Energ. 1997, 12, 117-122.

Wen, J.; Smith, T.F. “Absorption of solar energy in a room”. Solar Energy 2002, 72, 283-297.

Satoru, Y., Kensuke, A., Tamio, N., Hiroshi, K., Hisashi, A., "Novel humidity sensor using heat pipe: Phase transition thermally balanced sensor designed for measurement of humidity at high temperature", in Sensors and Actuators A: Physical, Volume 250, 15 2016, Pages 16.

Savchuk, Ol. A. Carvajal, J.J. Massons, J. Cascales, C. Aguiló, M. Díaz, F. "Novel low-cost, compact and fast signal processing sensor for ratiometric luminescent nanothermometry", in Sensors and Actuators A: Physical, Volume 250, 2016, Pages 87-95.

Schlapbach, L. Zuttel, A. "Hydrogen-storage materials for mobile applications".Nature 414 (2001) p. 353-358.

Shaowu Pan1, Zhitao Zhang, Wei Weng, Huijuan Lin, Zhibin Yang, Huisheng Peng. "Miniature wire-shaped solar cells, electrochemical capacitors and lithium-ion batteries" Vol. 17 Issue 6 (2014) p. 276-284.

Shuo Li; Jun Peng; Weirong Liu; Fu Jiang; Zhengfa Zhu; Kuo-Chi Lin. "Lifetime enhancement in wireless sensor and actuator network using uniform energy consumption algorithm". Int. J. of Embedded Systems, 2014 Vol.6, No.2/3, pp.233 - 239.
Simjee, F. and Chou, P. H. "Everlast: long-life, supercapacitor-operated wireless sensor node," in ISLPED '06: Proceedings of the 2006 international symposium on Low power electronics and design, (New York, NY, USA), pp. 197-202, ACM Press, 2006.

Xihai Zhang; Tianjian Wang; Junlong Fang. "A node localisation approach based on mobile beacon using particle swarm optimisation in wireless sensor networks". Int. J. of Embedded Systems, 2017 Vol.9, No.2, pp.112 - 118

Wang, W. Xuemei Sun, Wei Wu, Huisheng Peng, Yanlei Yu. "Photoinduced Deformation of Crosslinked Liquid-Crystalline Polymer Film Oriented by a Highly Aligned Carbon Nanotube Sheet" Angewandte Chemie International Edition. 51 (2012) 4644-4647.

Ward, J. A. Bharatula, N. B., Lukowicz, P. and Tröster, G. "Maximum power point tracking for on-body context systems.," in IEEE International Symposium on Wearable Computers (ISWC 2006), Switzerland, 2006.

Wilson, Jon. Sensor Technology Handbook. Newnes (imprint of Elsevier) 2005.

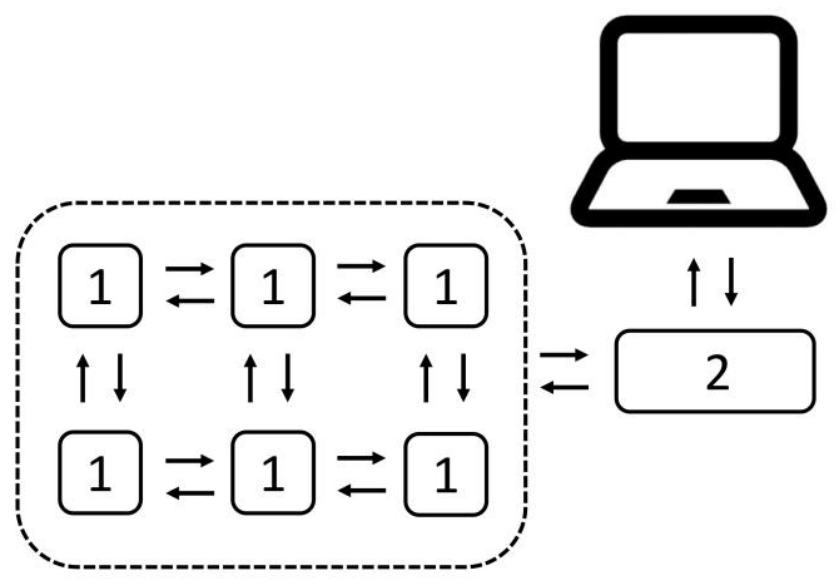

a) 


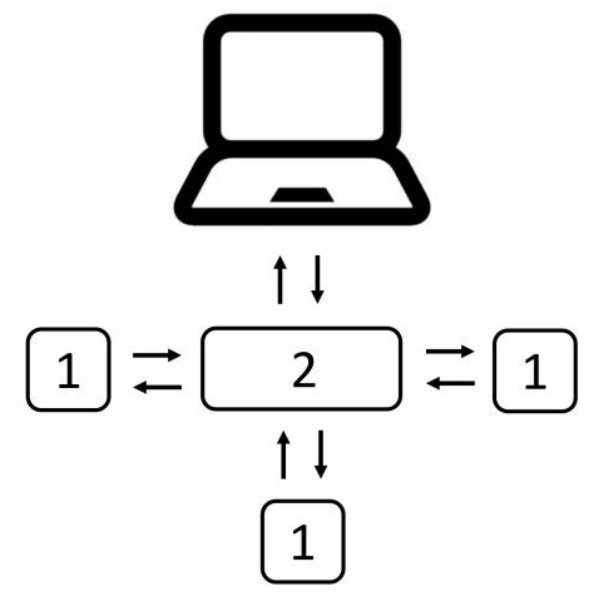

b)

Figure 1. a) wireless sensors (1) communicate with each other and with the receiver (2)-, b) star network system.

(5)

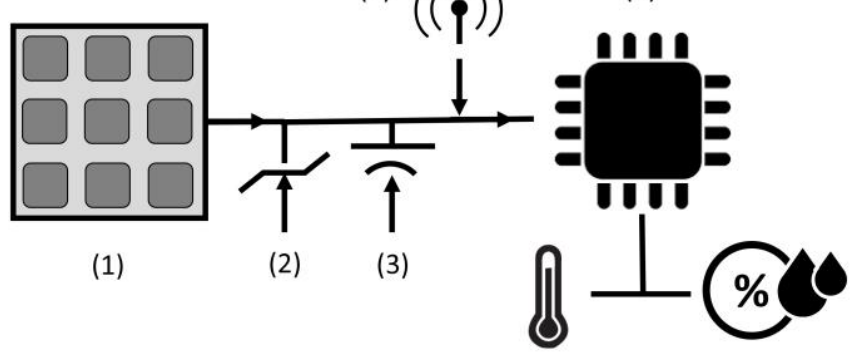

(6)

(7)

Figure 2. Description of the device. (1) PV microcell; (2) Zener; (3) Ultra Capacitor; (4); Radio frequency transmissor (5); Microcontroller; (6) Temperature Sensor; (7) Relative hunidity Sensor.

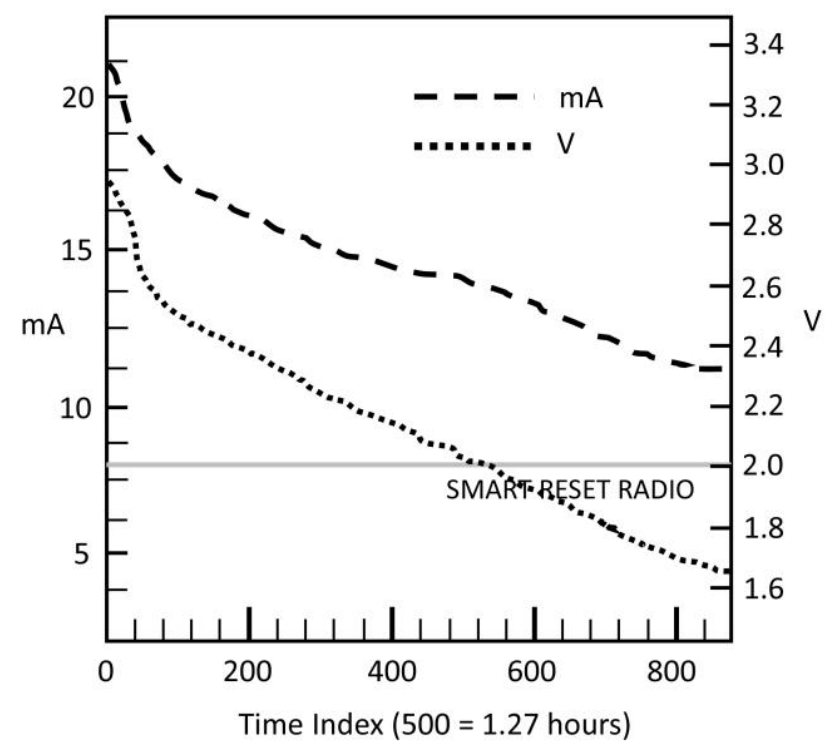

a)

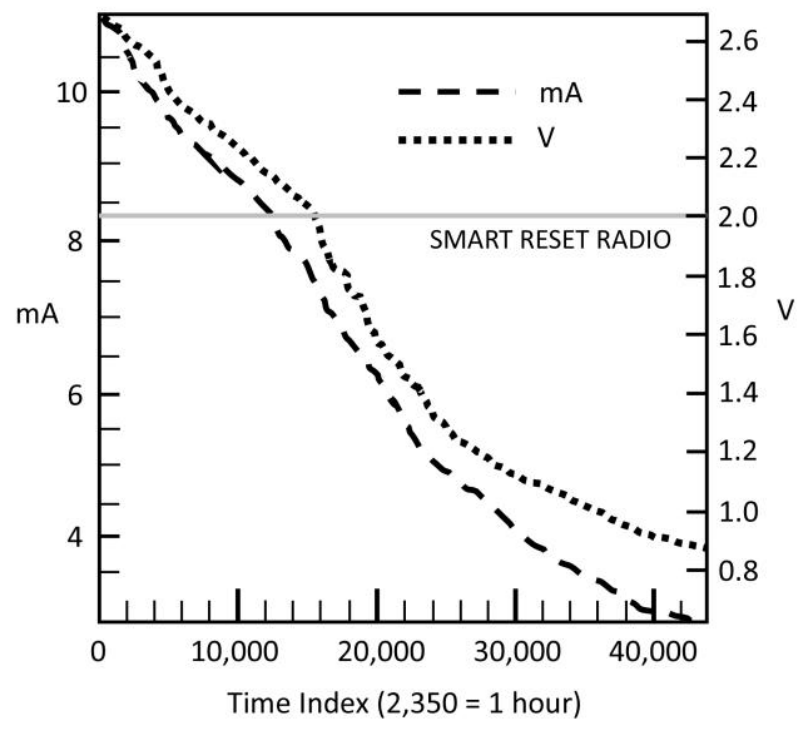

b)

Figure 3. I, V curves using battery, a) resistor at $20 \mathrm{~mA}$; b) resistor at 13.6 $\mathrm{mA}$.

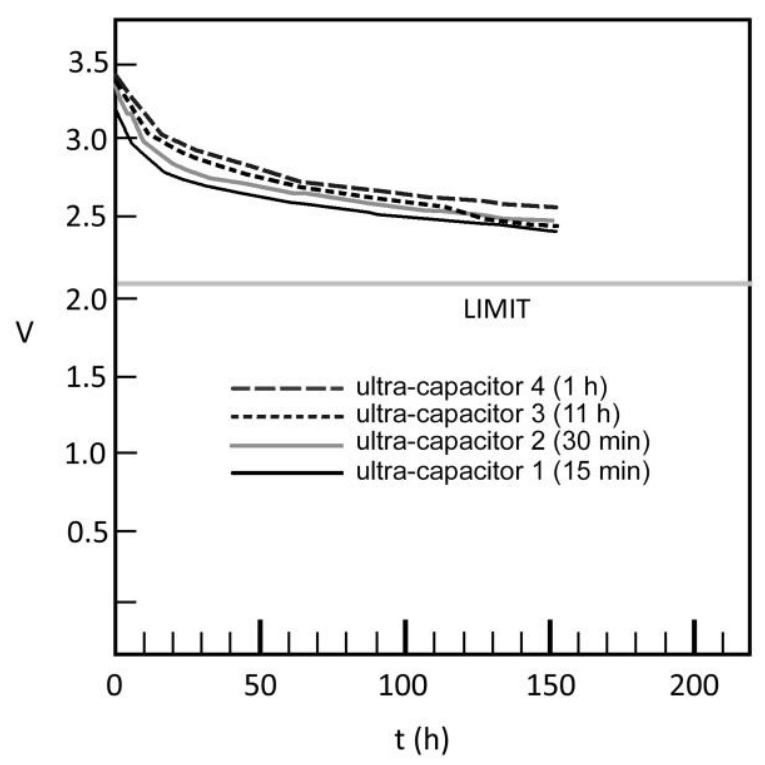

Figure 4. Comparison of four different ultra-capacitors. Self descharge test.

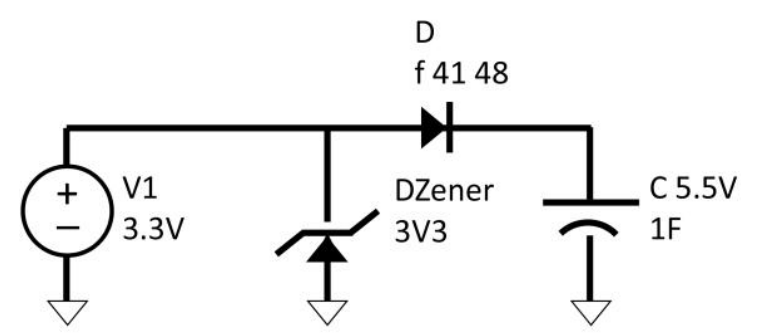

Figure 5. Block diagram of the testing devices. 


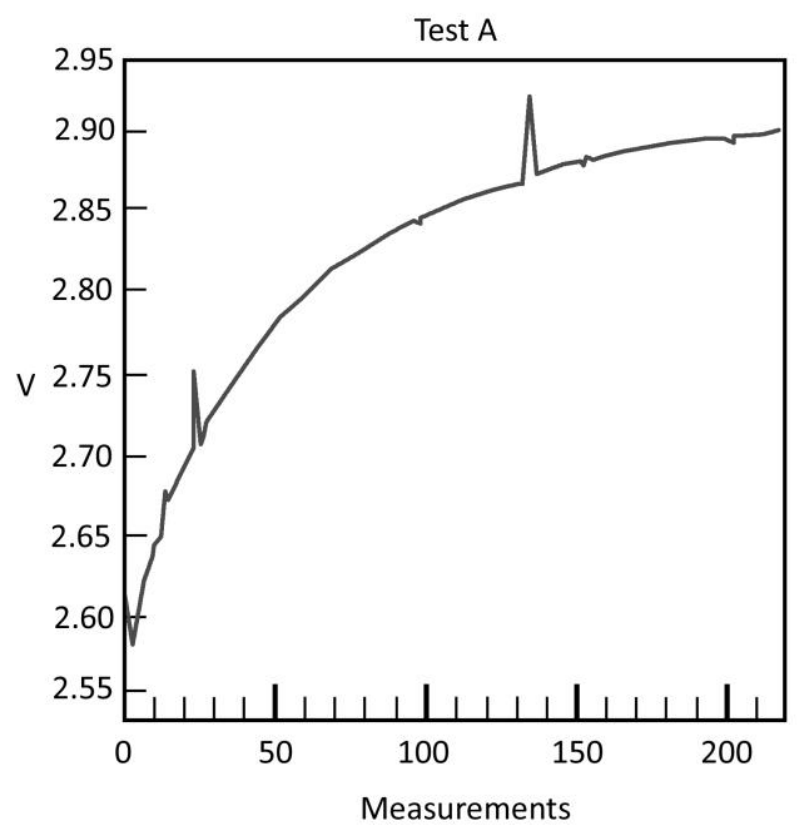

a)

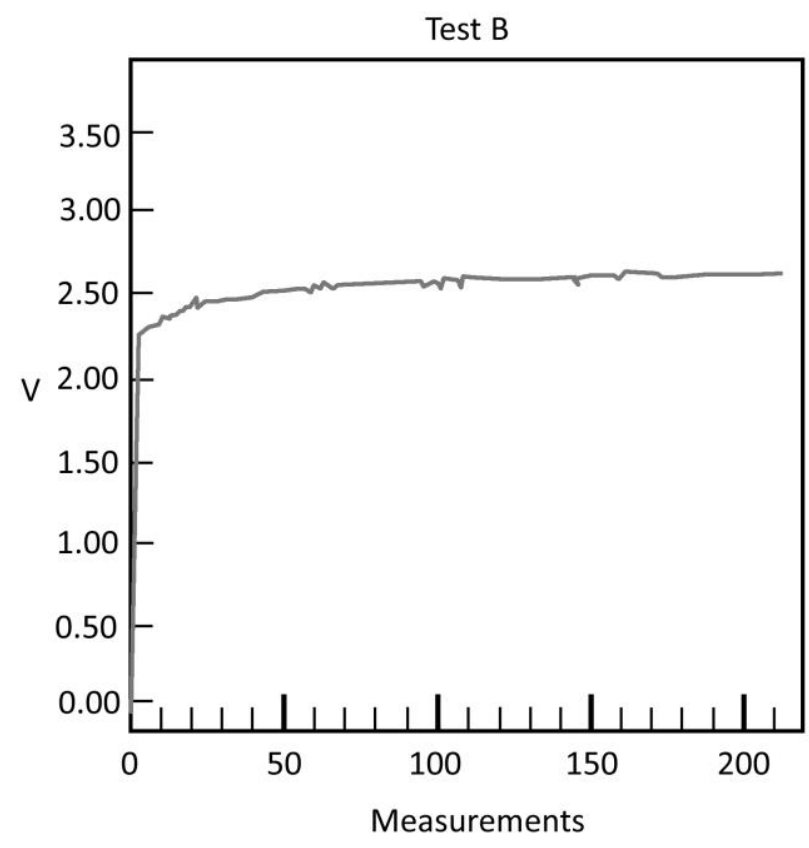

b)

Figure 6. Charging time of ultra-capacitor using zener and diode; a) test A voltage $=3.364 \mathrm{~V} ; \mathrm{b}$ ) test $\mathrm{B}$ voltage $=3.034 \mathrm{~V}$.

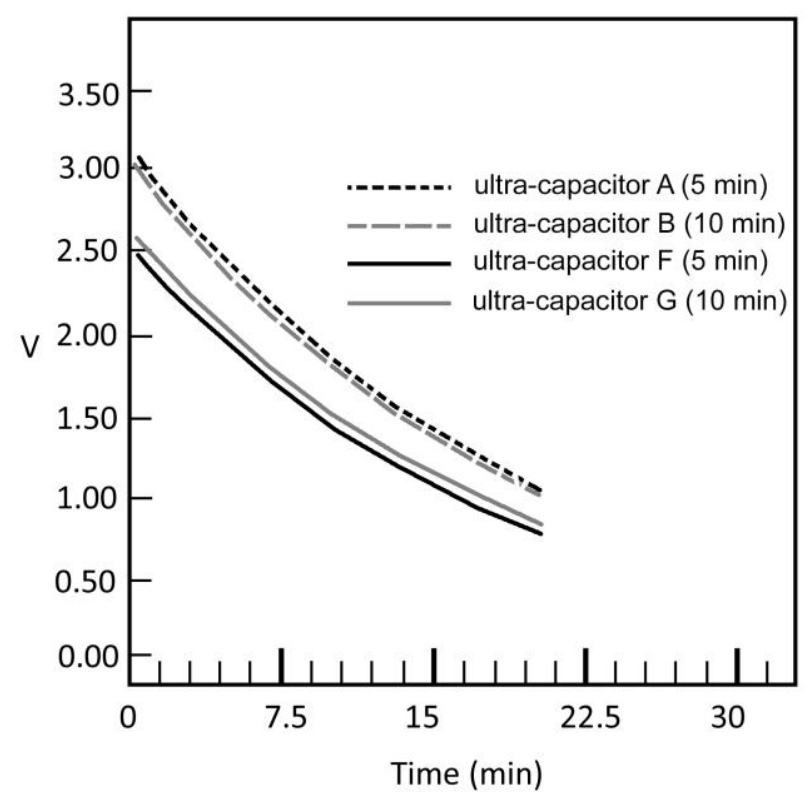

Figure 7. Comparison among Resistor Discharge Ultra Capacitors. $\mathrm{R}=1000 \Omega, \mathrm{VF}=3.320 \mathrm{~V}$

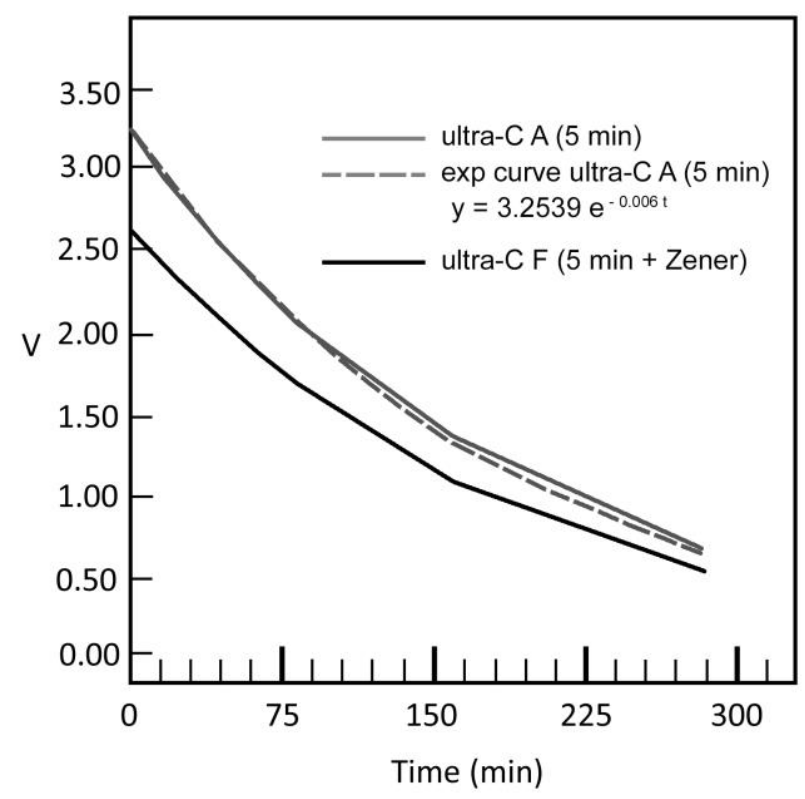

Figure 8. Resistor Discharge Ultra-Capacitor. $\mathrm{R}=11000 \Omega, \mathrm{V}_{\mathrm{F}}=3.331 \mathrm{~V}$ 


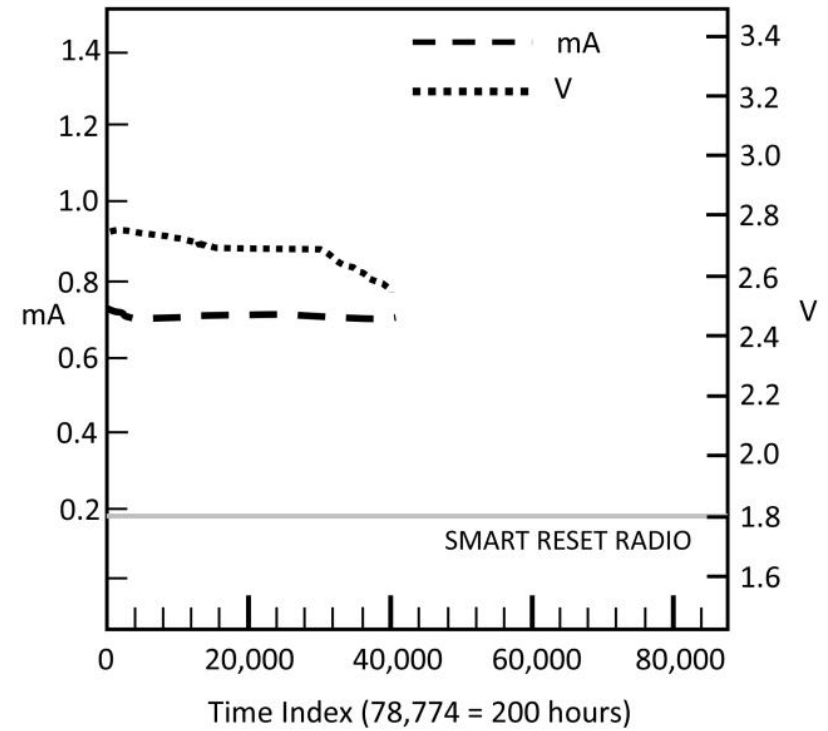

a) Data every $100 \mathrm{~ms}$

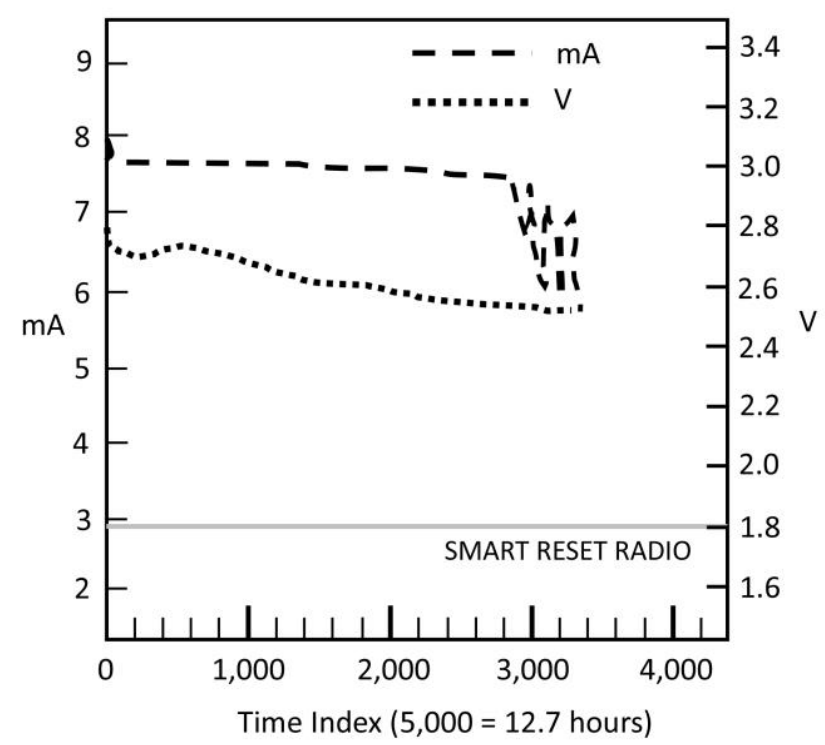

b) Data every $10 \mathrm{~ms}$

Figure 9. I, V curves using battery and capacitor; a) sending data every 100 $\mathrm{ms}$; b) sending data every $10 \mathrm{~ms}$.

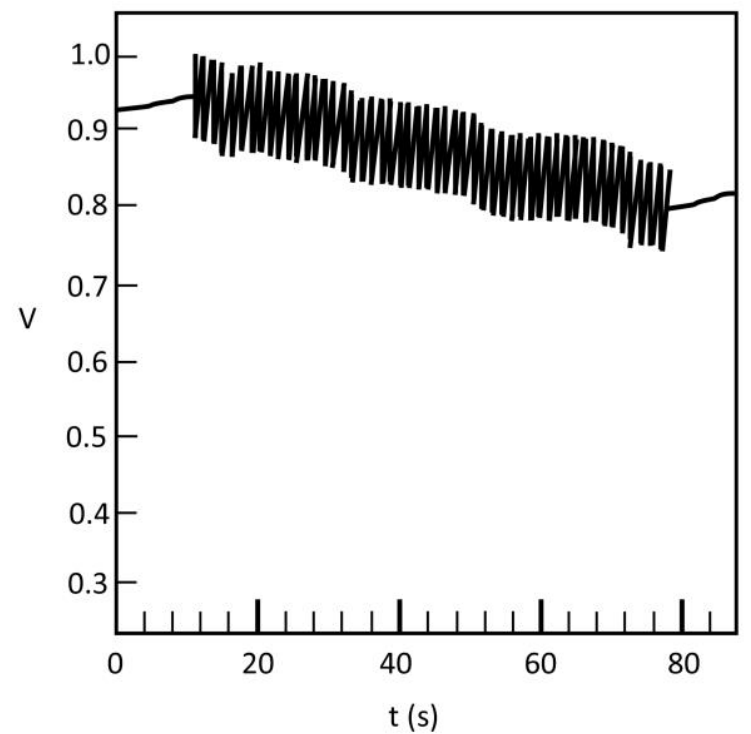

a) Without incident light

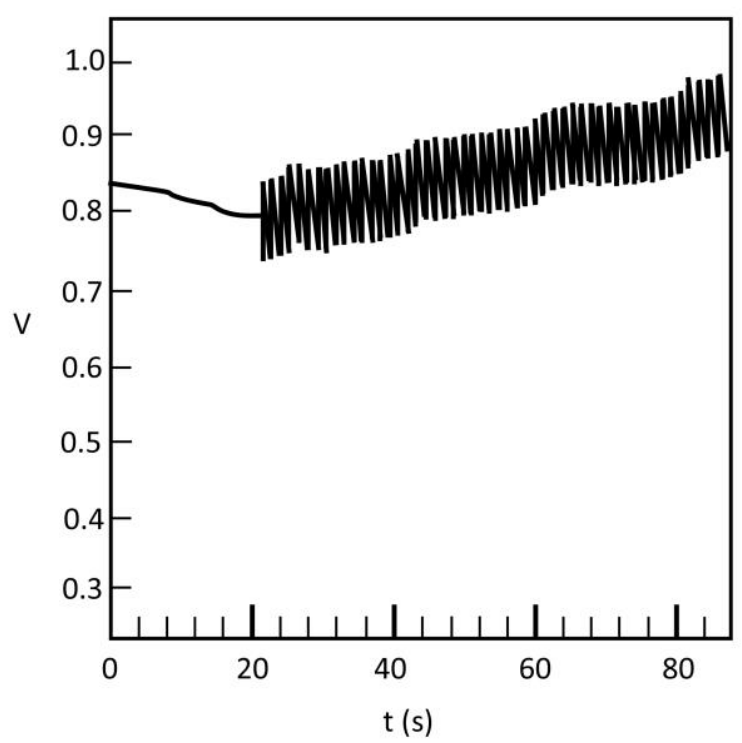

b) With incident light

Figure 10. Voltage level of the ultracapacitor used as energy reservoir a) without incident light; b) with incident light. 